\title{
Aspirin may inhibit angiogenesis and induce autophagy by inhibiting mTOR signaling pathway in murine hepatocarcinoma and sarcoma models
}

\author{
QIANQIAN ZHAO ${ }^{1,2}$, ZHAOPENG WANG $^{1}$, ZHAOXIA WANG $^{1}$, LICUN WU $^{3}$ and WEIDONG ZHANG ${ }^{1}$ \\ ${ }^{1}$ Key Laboratory for Modern Medicine and Technology of Shandong, Institute of Basic Medicine; \\ ${ }^{2}$ School of Medicine and Life Sciences, University of Jinan, Shandong Academy of Medical Sciences, Jinan, \\ Shandong 250062, P.R. China; ${ }^{3}$ Latner Thoracic Surgery Research Laboratories and Division of Thoracic Surgery, \\ Toronto General Hospital, University Health Network, University of Toronto, Toronto, ON M5G 1L7, Canada
}

Received May 6, 2015; Accepted June 16, 2016

DOI: $10.3892 / \mathrm{ol} .2016 .5017$

\begin{abstract}
Aspirin is known to have inhibitory effects on growth development in various types of tumor. In previous studies, it was observed to inhibit angiogenesis by downregulating the expression of vascular endothelial growth factor-A (VEGF-A). In the present study, murine $\mathrm{H}_{22}$ hepatocarcinoma and S180 sarcoma models were used to ascertain whether aspirin could inhibit angiogenesis and promote autophagy in tumors. Tumor-bearing mice were randomly divided into four groups with 10 mice per group: i) no treatment; ii) low-dose aspirin (100 mg/kg); iii) high-dose aspirin (400 mg/kg); iv) everolimus group ( $4 \mathrm{mg} / \mathrm{kg}$ ). The effects of high-dose aspirin were validated through preliminary experiments. The drug treatment was administered every day for 14 days. The tumor size was measured every other day and then the tumor growth curve was plotted, and the tumor inhibitory rates were calculated. The expression levels of phosphorylated mammalian target of rapamycin (p-mTOR),
\end{abstract}

Correspondence to: Professor Weidong Zhang, Key Laboratory for Modern Medicine and Technology of Shandong, Institute of Basic Medicine, Shandong Academy of Medical Sciences, 18877 Jingshi Road, Jinan, Shandong 250062, P.R. China

E-mail: zhangweidongkui@163.com

Dr Licun Wu, Latner Thoracic Surgery Research Laboratories and Division of Thoracic Surgery, Toronto General Hospital, University Health Network, University of Toronto, 101 College Street, Toronto, ON M5G 1L7, Canada

E-mail: wulicun@hotmail.com

Abbreviations: mTOR, mammalian target of rapamycin; p-mTOR, phosphorylated mammalian target of rapamycin; HIF- $1 \alpha$, hypoxia-inducible factor-1 $\alpha$; VEGF-A, vascular endothelial growth factor-A; ULK1, UNC-51-like kinase-1; LC3A, microtubule-associated protein 1 light chain $3 \mathrm{~A}$

Key words: mammalian target of rapamycin, autophagy, angiogenesis, tumor, aspirin hypoxia-inducible factor-1 $\alpha$ (HIF-1 $\alpha$ ), VEGF-A, UNC-51-like kinase-1 (ULK1) and microtubule-associated protein 1 light chain 3A (LC3A) were detected by immunohistochemistry and western blot analysis, respectively. We observed that tumor growth delay was achieved in both $\mathrm{H}_{22}$ hepatocarcinoma and S180 sarcoma models following treatment with aspirin. The tumor growth inhibition rates induced by low and high-dose aspirin and everolimus were 19.6, 33.6 and $53.7 \%(\mathrm{P}<0.05)$ in $\mathrm{H}_{22}$ hepatocarcinoma, and 25.7, 40.6 and $48.7 \%(\mathrm{P}<0.05)$ in S180 sarcoma. The immunohistochemistry and western blot analysis data from the models revealed that the expression of p-mTOR, HIF-1 $\alpha$ and VEGF-A was decreased, while the expression of ULK1 and LC3A was increased following treatment with aspirin and everolimus. The changes were more apparent in the high-dose aspirin and everolimus groups $(\mathrm{P}<0.01)$. The inhibitory action of aspirin and everolimus on tumor angiogenesis may be through inhibiting the expression of p-mTOR, HIF-1 $\alpha$ and VEGF-A. Alternatively, aspirin may induce autophagy by inhibiting the mTOR signaling target and then increasing ULK1 and LC3A.

\section{Introduction}

Aspirin, a nonsteroidal anti-inflammatory drug, is used clinically as antipyretic, analgesic and anti-inflammatory medicine. It has been indicated to reduce the risk of cancers, including bladder cancer (1), breast cancer (2), glioma (3), and particularly colorectal cancer $(4,5)$. Although the considerable evidence demonstrating that aspirin prevents cancer progression is compelling, the underlying molecular mechanism remains enigmatic. Numerous molecular targets and pathways have been implicated; however, the antitumor activity of aspirin may be not attributed wholly to a single target or pathway (6). It is likely that aspirin influences several molecular pathways that cross each other.

Mammalian target of rapamycin (mTOR) is a $289-\mathrm{kDa}$ serine/threonine kinase which is highly expressed in the processes of multiple types of tumors as the control center of cell growth, differentiation, apoptosis and angiogenesis $(7,8)$. In mammalian cells, mTOR-dependent processes include 
regulating cell growth by controlling mRNA translation, ribosome biogenesis, autophagy and metabolism $(9,10)$.

Angiogenesis, the formation of new blood vessels, is required for tumor growth and metastasis. Several regulating pathways have been involved in this process. Through immunohistochemistry and western blot analysis, we observed that hypoxia-inducible factor- $1 \alpha(\mathrm{HIF}-1 \alpha)$ and vascular endothelial growth factor-A (VEGF-A) are the downstream proteins of mTOR. Tumor tissue is usually accompanied by hypoxia, which promotes HIF-1 $\alpha$ production. HIF-1 $\alpha$ and its downstream target, VEGF-A, play critical roles in tumor angiogenesis and represent an attractive chemotherapeutic target (11-13). Ruan et al revealed that the phosphatidylinositol 3-kinase (PI3K)/AKT/mTOR signaling pathway as 'the regulation center of angiogenesis' could regulate the expression of VEGF-A by hypoxia and HIF-1 $\alpha$, cancer genes, hormones, growth factors and cytokines and other factors (14). Another study also revealed that although mTOR activity was restrained in hypoxia, the cells still mediated the production of HIF-1 $\alpha$ through the mTOR pathway (15). Under normoxia, improving the activity of mTOR increases the expression of HIF-1 $\alpha$ in tumor cells (16).

Autophagy is an evolutionarily conserved process in which cells recycle long-lived proteins and damaged organelles. It involves the sequestration of cytoplasmic components within a double membrane structure, called autophagosome, and subsequent delivery to lysosomes for degradation $(17,18)$. Atg1, with its mammalian homologue UNC-51-like kinase-1 (ULK1), is a conserved serine-threonine kinase that is required for autophagy pathways, and its activity is regulated by the TOR kinase (19-21). Yeast Atg8, and its mammalian homolog microtubule-associated protein 1 light chain 3 (LC3), are ubiquitin-like modifiers that are localized on isolation membranes and play crucial roles in the formation of autophagosomes. Yeast expresses a single Atg8 protein, while mammals encode several isoforms, including three MAP1 light chain 3 proteins [LC3A (two splice variants), B and C)] and four $\gamma$-aminobutyrate receptor-associated proteins (22). mTOR exists in a phosphorylated form in normal conditions and suppresses autophagy. However, when the phosphorylated mTOR (p-mTOR) level is downregulated, as observed during rapamycin treatment or nutrient starvation, cell autophagy is induced (23).

Downregulation of the mTOR pathway due to treatment with mTOR inhibitors suppresses tumor angiogenesis and enhances autophagy $(24,25)$. Aspirin inhibits mTOR signaling in colorectal cancer and angiogenesis in murine sarcoma models $(6,26)$. Based on this evidence, experimental studies using $\mathrm{H}_{22}$ hepatocarcinoma and S180 sarcoma models were designed to investigate the underlying mechanisms of the antitumor effects of aspirin.

\section{Materials and methods}

Materials. Aspirin, purchased from Qilu Pharmaceutical Co., Ltd. (Shandong, China), was dissolved from powder into drinking water and stored at $4^{\circ} \mathrm{C}$. Everolimus was purchased from Ruibio (Sachsen, Germany) and an original concentration of $40 \mathrm{mM}$ was prepared with dimethyl sulfoxide (DMSO) as a stock solution at $4^{\circ} \mathrm{C}$. DMSO was obtained from
Sigma-Aldrich (St. Louis, MO, USA). A bicinchoninic acid (BCA) protein assay kit was obtained from Pierce (Rockford, IL, USA). Polyvinylidene difluoride (PVDF) membranes were from Pall Life Sciences (Ann Arbor, MI, USA). Western blot-related reagents were purchased from the Shanghai Beyotime Institute of Biotechnology, China.

Animal models. The animal experiments were approved by the Institute of Medicine, Shandong Academy of Medical Sciences, China. Forty male Kunming mice aged 5-6 weeks and weighing 18-22 g were obtained from the Animal Experiment Center of Shandong University, China. Under sterile conditions, ascites were extracted from $\mathrm{H}_{22}$ ascitic mice which had been injected intraperitoneally with $\mathrm{H}_{22}$ cells seven days previously. Normal saline was added to adjust the cell concentration to $1 \times 10^{7} / \mathrm{ml}$. Then $0.2 \mathrm{ml}$ tumor cell suspension was inoculated subcutaneously into the right flank of each mouse, and the S180 sarcoma model was processed in the same way. The mice were maintained under standard husbandry conditions and monitored for body weight and water and food intake.

Drug treatment. Following tumor cell injection, the mice were randomly divided into four groups with ten mice in each group when the tumors were $\sim 50-100 \mathrm{~mm}^{3}$. The four groups were as follows: i) no treatment, used as control; ii) low-dose aspirin (100 mg/kg); iii) high-dose aspirin (400 mg/kg); iv) everolimus group ( $4 \mathrm{mg} / \mathrm{kg}$ ). The control group took in purified water. Aspirin with was administered by oral gavage once daily for 14 days. Everolimus was administered by oral gavage at $4 \mathrm{mg} / \mathrm{kg}$ every day for 14 days. Tumor size was measured every other day using a digital caliper, and tumor volume was calculated using the formula: $\mathrm{V}=\mathrm{AxB}^{2} / 2$, where $\mathrm{A}$ and $\mathrm{B}$ are the largest diameter and the perpendicular one, respectively. The body weight of the mice was measured every day and the experiment lasted 3 weeks. At the end of the experiment, blood was taken by removing the eyeball and the tumors were dissected and weighed after euthanasia. The tumor inhibitory rate was calculated using the formula: Inhibitory rate $($ IR) $=$ [average tumor weight of control group $(g)$ - average tumor weight of intervention group $(\mathrm{g})]$ / average tumor weight of control group $(\mathrm{g}) \times 100 \%$. Sections of each tumor were immediately placed in $4 \%$ paraformaldehyde for immunohistochemistry. The remainder of each tumor was snap frozen for western blotting and stored at $-70^{\circ} \mathrm{C}$ until processing.

Histology and immunohistochemistry. Tumor tissue was treated by paraffin embedding after being placed overnight in $4 \%$ paraformaldehyde. The paraffin blocks within the tumor tissue were cut into $4-\mu \mathrm{m}$ sections for immunohistochemical staining. Sections were de-waxed in xylene, then rehydrated through ethanol divided into different gradients and rinsed by distilled water. Following treatment with $3 \%$ hydrogen peroxide in absolute methanol to inactivate endogenous peroxidase activity, sections were subjected to hot repair with citrate antigen retrieval solution ( $\mathrm{pH} \mathrm{6.0)}$ for $15 \mathrm{~min}$ to expose epitope, and then cooled to room temperature. Sections were washed with phosphate-buffered saline (PBS) three times, five minutes each time, then the primary antibody was added and subsequently incubated overnight at $4^{\circ} \mathrm{C}$. The sections were incubated at room temperature for $50 \mathrm{~min}$ and then 
washed again with PBS three times and sequentially incubated with the secondary antibody. The tissue was incubated at room temperature for $1 \mathrm{~h}$, and then washed with PBS and colored with 3,3'-diaminobenzidine for 2-10 min and finally counterstained with hematoxylin.

Western blotting. The expression profiles for p-mTOR, HIF-1 $\alpha$, VEGF-A, ULK1 and LC3A were determined by western blot analysis. The tumor tissue stored at $-70^{\circ} \mathrm{C}$ was sheared, ground, centrifugated and boiled. The protein concentration was determined with the BCA kit and then the samples were stored at $-20^{\circ} \mathrm{C}$. Equal quantities $(20 \mu \mathrm{g})$ of samples from different groups were loaded onto sodium dodecyl sulphate-polyacrylamide electrophoresis gel and the resolved proteins were electrotransferred to PVDF membranes. Membranes were sealed with 5\% skimmed milk in TBST (1 M Tris-buffered saline, $\mathrm{pH}$ 7.6; $5 \mathrm{M} \mathrm{NaCl}$; $0.1 \%$ Tween-20) buffer for $2 \mathrm{~h}$ before adding primary antibody, and incubated overnight at $4^{\circ} \mathrm{C}$. Western blot analyses were carried out using the appropriate antibody [p-mTOR, LC3A (Cell Signaling Technology, Inc., Danvers, MA, USA); ULK1 (Abcam, Hong Kong, China); HIF-1 $\alpha$, VEGF-A (Beijing Biosynthesis Biotechnology Co., Ltd., Beijing, China)]. The membranes were then developed using the ECL Plus chemiluminescence detection system. The band intensities were analyzed by ImageJ software (Wayne Rasband National Institutes of Health, Bethesda, MD, USA) and normalized to $\beta$-actin (Cell Signaling Technology, Inc.).

Statistical analysis. The descriptive statistics provided are the means \pm standard deviation. A one-way analysis of variance (ANOVA) test was used to assess the effects of different doses for aspirin and everolimus on tumor tissues. Data were analyzed using an ANOVA pairwise comparison method (Bonferroni method) and the Spearman rank correlation analysis. $\mathrm{P}<0.05$ was considered to indicate a statistically significant difference.

\section{Results}

Effects of aspirin on $\mathrm{H}_{22}$ hepatoma tumor growth. In the control and drug intervention groups, tumor dimensions increased. Compared with the control group, the everolimus group $(4 \mathrm{mg} / \mathrm{kg})$, high-dose aspirin group $(400 \mathrm{mg} / \mathrm{kg})$ and low-dose aspirin $(100 \mathrm{mg} / \mathrm{kg})$ group markedly inhibited tumor growth (Table I, Fig. 1). The inhibitory rates were 53.7, 36.7 and $21.1 \%$, respectively $(\mathrm{P}<0.05$ for each comparison between treatment groups and the control group). The inhibitory effects were more apparent in the high-dose aspirin group and the everolimus group $(\mathrm{P}<0.01)$. Although the inhibitory rate of the low-dose aspirin group was lower, the mice in this group were generally in good condition, with a food and drink intake and weight similar to those of mice in the high-dose aspirin group. This suggests that aspirin may improve the living standard of tumor-bearing mice.

Expression of p-mTOR, HIF-1 $\alpha, V E G F-A, U L K 1$ and LC3A in $\mathrm{H}_{22}$ hepatocarcinoma tumors. Based on the immunohistochemical staining results, p-mTOR, ULK1 and VEGF-A were expressed in the cytoplasm of tumor cells. HIF-1 $\alpha$ was expressed in the nucleus and cytoplasm of tumor cells. LC3A was expressed in the cytoplasm or membrane. Cells positive
Table I. Inhibitory effect of aspirin on $\mathrm{H}_{22}$ hepatocarcinoma (mean \pm standard deviation, $\mathrm{n}=10$ ).

\begin{tabular}{lcc}
\hline Group & Tumor weight (g) & IR (\%) \\
\hline Control & $1.47 \pm 0.21$ & - \\
Low-dose aspirin & $1.16 \pm 0.19^{\mathrm{a}}$ & 21.1 \\
High-dose aspirin & $0.93 \pm 0.14^{\mathrm{b}}$ & 36.7 \\
Everolimus & $0.68 \pm 0.12^{\mathrm{b}}$ & 53.7 \\
\hline
\end{tabular}

Control, treated with normal saline; IR, inhibitory rate; ${ }^{\mathrm{a}} \mathrm{P}<0.05$, ${ }^{\mathrm{b}} \mathrm{P}<0.01$, vs. control.

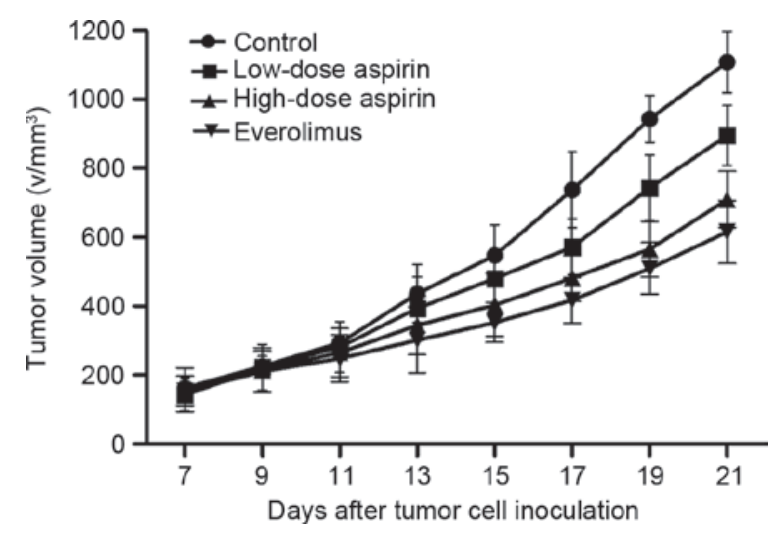

Figure 1. Tumor growth curves of $\mathrm{H}_{22}$ hepatocarcinoma following tumor cell inoculation. There were 10 mice per group, under the same experimental conditions. Treatment was initiated at day 7 following tumor cell inoculation. Control, treated with normal saline.

for p-mTOR, HIF-1 $\alpha$, VEGF-A, ULK1 and LC3A were stained brown (Fig. 2). The expression of p-mTOR, HIF-1 $\alpha$ and VEGF-A in the control group was markedly higher than that in the treatment groups. The expression of ULK1 and LC3A in the treatment groups was higher than that in the control group, particularly in the everolimus and high-dose aspirin groups. Gray scale intensity variants of p-mTOR, HIF-1 $\alpha$, VEGF-A, ULK1 and LC3A immunoreactivity were evaluated by Leica Qwin V3 software (Leica Microsystems GmbH, Wetzlar, Germany). Five positive regions selected randomly from each section were analyzed at an original magnification of $\mathrm{x} 400$. An inverse correlation was observed between the gray scale intensity and the protein expression: the higher the gray scale intensity, the weaker the protein expression, and vice versa (Fig. 2). In each comparison, there was a significant difference $(\mathrm{P}<0.05)$. In addition, the high-dose aspirin and everolimus groups were significantly different compared with the control group $(\mathrm{P}<0.01)$. $\mathrm{p}$-mTOR, HIF-1 $\alpha$ and VEGF-A expression was positively correlated $(r=0.788, \mathrm{P}<0.01 ; \mathrm{r}=0.776, \mathrm{P}<0.01)$; and HIF- $1 \alpha$ and VEGF-A expression was positively correlated $(\mathrm{r}=0.796, \mathrm{P}<0.01)$. $\mathrm{p}-\mathrm{mTOR}$, ULK1 and LC3A expression was negatively correlated $(\mathrm{r}=-0.804, \mathrm{P}<0.01 ; \mathrm{r}=-0.703, \mathrm{P}<0.01)$; ULK1 and LC3A expression were positively correlated $(\mathrm{r}=0.734, \mathrm{P}<0.01)$.

Effect of aspirin treatment on p-mTOR, HIF-1 $\alpha, V E G F-A$, ULK1 and LC3A protein expression as assessed by western 
Table II. Inhibitory effect of aspirin on S180 sarcoma (mean \pm standard deviation, $\mathrm{n}=10$ ).

\begin{tabular}{lcc}
\hline Group & Tumor weight $(\mathrm{g})$ & IR $(\%)$ \\
\hline Control & $2.05 \pm 0.33$ & - \\
Low-dose aspirin & $1.57 \pm 0.38^{\mathrm{a}}$ & 23.4 \\
High-dose aspirin & $1.32 \pm 0.27^{\mathrm{a}}$ & 35.6 \\
Everolimus & $1.06 \pm 0.22^{\mathrm{a}}$ & 48.7 \\
\hline
\end{tabular}

Control, treated with normal saline; IR, inhibitory rate; ${ }^{a} \mathrm{P}<0.01 \mathrm{vs.}$ control.
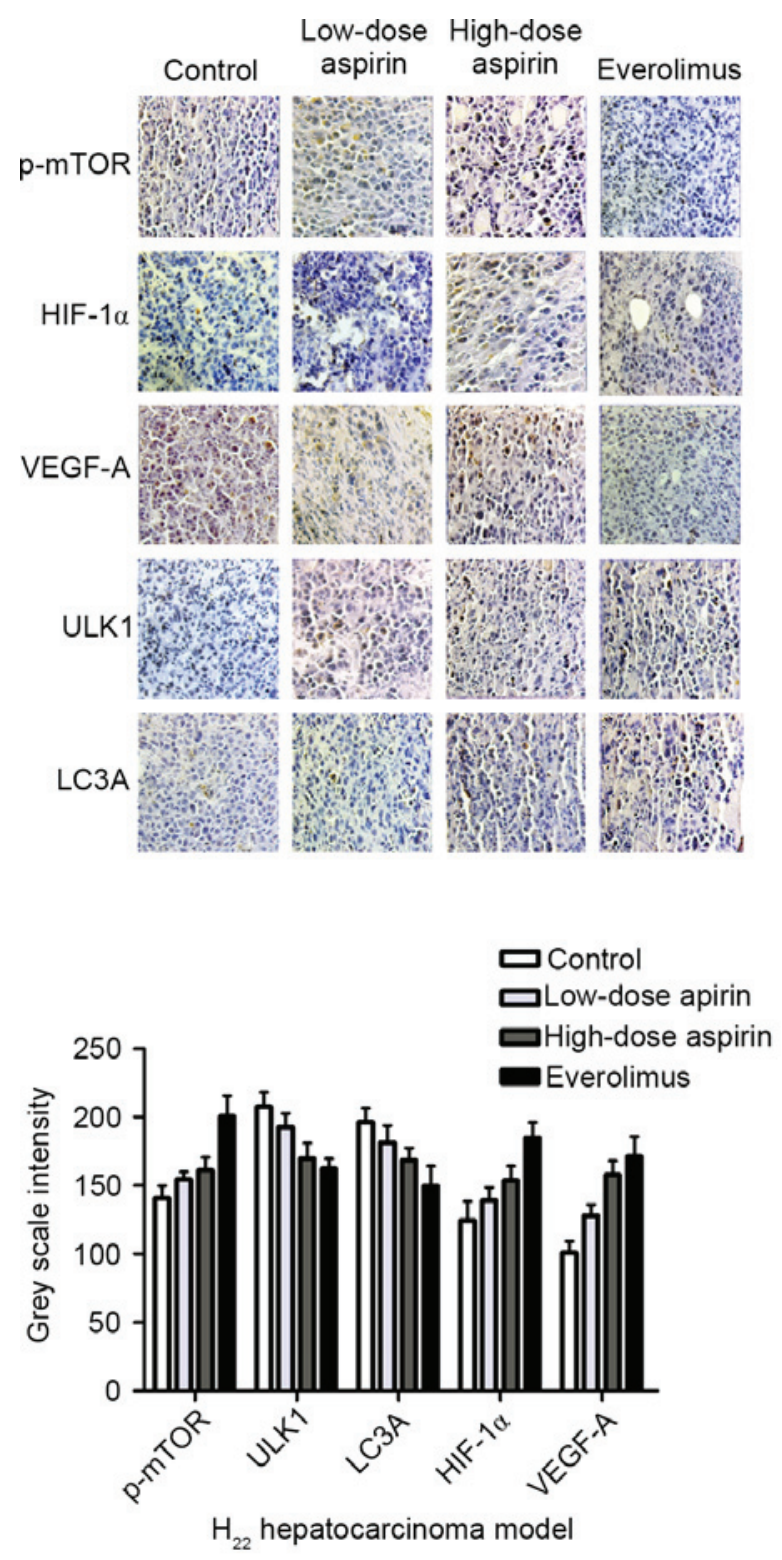

Figure 2. Expression of phosphorylated mammalian target of rapamycin (p-mTOR), hypoxia-inducible factor- $1 \alpha$ (HIF-1 $\alpha$ ), vascular endothelial growth factor-A (VEGF-A), UNC-51-like kinase-1 (ULK1) and microtubule-associated protein 1 light chain $3 \mathrm{~A}$ (LC3A) in $\mathrm{H}_{22}$ hepatocarcinoma tissue was detected by immunohistochemistry (original magnification, $\mathrm{x} 400$ ). Gray scale intensity variants were evaluated by Leica Qwin V3 software. Sections were taken from five randomly selected positive regions (original magnification, $\mathrm{x} 400$ ). Higher gray scale intensity represents weaker protein expression, and lower intensity represents stronger protein expression. The three treatment groups are significantly different compared with the control, $\mathrm{P}<0.05$.
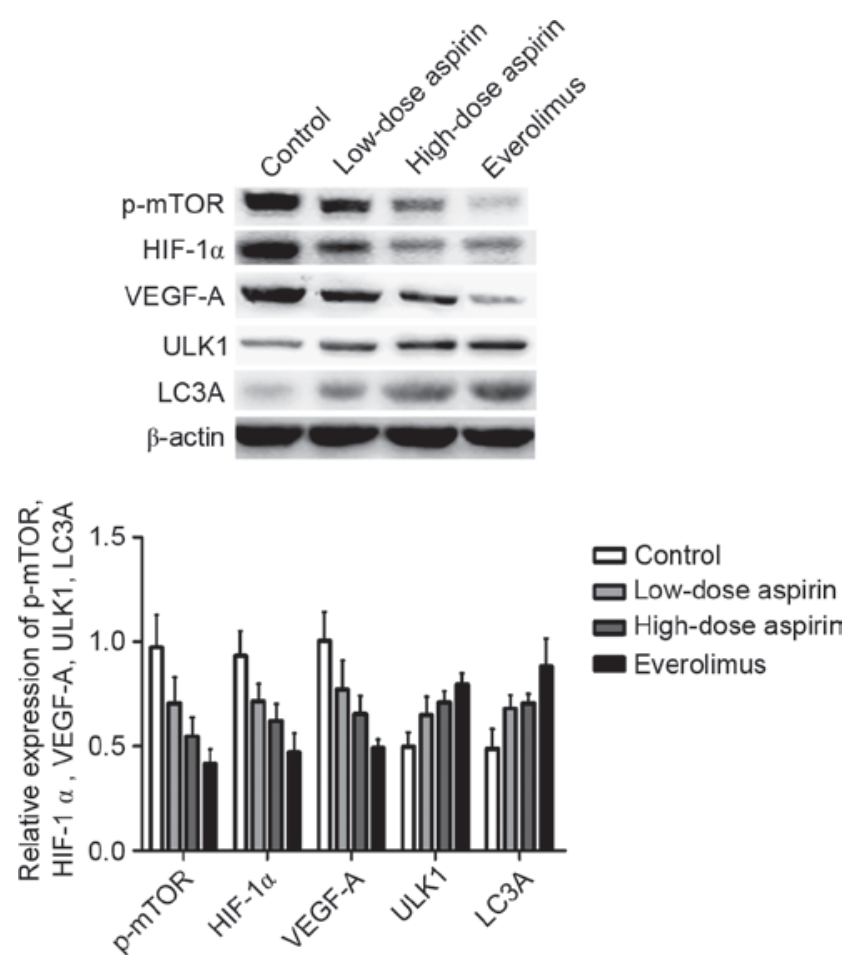

Figure 3. Effects of aspirin on expression of phosphorylated mammalian target of rapamycin (p-mTOR), hypoxia-inducible factor- $1 \alpha$ (HIF-1 $\alpha$ ), vascular endothelial growth factor-A (VEGF-A), UNC-51-like kinase-1 (ULK1) and microtubule-associated protein 1 light chain $3 \mathrm{~A}$ (LC3A) in $\mathrm{H}_{22}$ hepatocarcinoma tissue were detected by western blot analysis. The relative expression of p-mTOR, HIF-1 $\alpha$, VEGF-A, ULK1 and LC3A was analyzed by ImageJ software. In each comparison, there was a significant difference $(\mathrm{P}<0.05)$.

blot analysis in $\mathrm{H}_{22}$ hepatocarcinoma tumors. P-mTOR, HIF-1 $\alpha$, VEGF-A, ULK1 and LC3A expression was normalized to $\beta$-actin expression by band intensity. As shown in Fig. 3, the expression of p-mTOR, HIF- $1 \alpha$ and VEGF-A decreased in the low-dose and high-dose aspirin and everolimus groups, but the expression of ULK1 and LC3A increased when compared with the control group in the $\mathrm{H}_{22}$ hepatocarcinoma tissue. Band intensities were analyzed using ImageJ software. The expression demonstrated a dose-dependent trend in the high-dose and low-dose aspirin groups. In addition, aspirin at each concentration demonstrated a significant difference among groups $(\mathrm{P}<0.05)$. Furthermore, $\mathrm{p}$-mTOR, HIF-1 $\alpha$ and VEGF-A expression was positively correlated $(\mathrm{r}=0.845$, $\mathrm{P}<0.01 ; \mathrm{r}=0.802, \mathrm{P}<0.01)$; HIF- $1 \alpha$ and VEGF-A expression was positively correlated $(\mathrm{r}=0.856, \mathrm{P}<0.01)$; $\mathrm{p}$-mTOR, ULK1 and LC3A expression was negatively correlated $(\mathrm{r}=-0.528$, $\mathrm{P}<0.05 ; \mathrm{r}=-0.636, \mathrm{P}<0.01)$; and ULK1 and LC3A expression was positively correlated $(\mathrm{r}=0.779, \mathrm{P}<0.01)$. $\mathrm{P}-\mathrm{mTOR}$ expression also demonstrated a dose-dependent trend in the high-dose and low-dose aspirin groups. In addition, aspirin at each concentration was significantly different between groups $(\mathrm{P}<0.05)$.

Effect of aspirin on S180 sarcoma tumor growth. The experimental conditions and the processing method of the S180 sarcoma model was the same as that used for $\mathrm{H}_{22}$ tumors. Data were analyzed using an ANOVA pairwise comparison method (Bonferroni method). The average tumor dimension of each group was larger and the inhibitory rates were 48.7, 


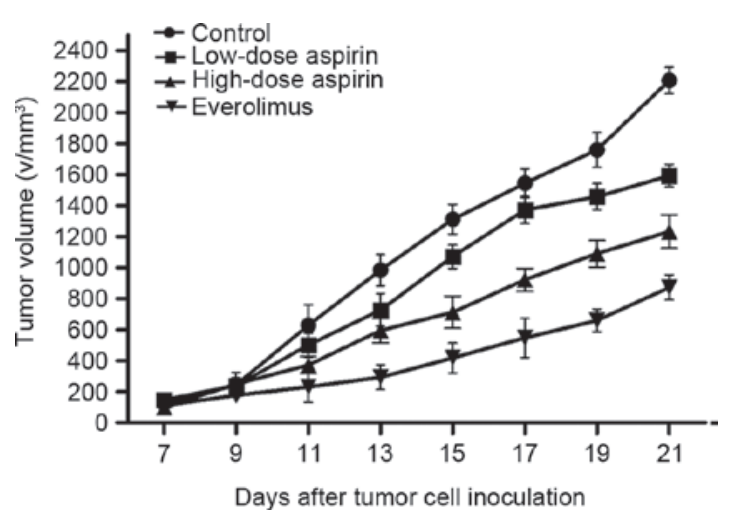

Figure 4. Growth curves of S180 sarcoma following tumor cell inoculation. There were 10 mice per group, under the same experimental conditions. Treatment was initiated at day 7 following tumor cell inoculation. Control, treated with normal saline.
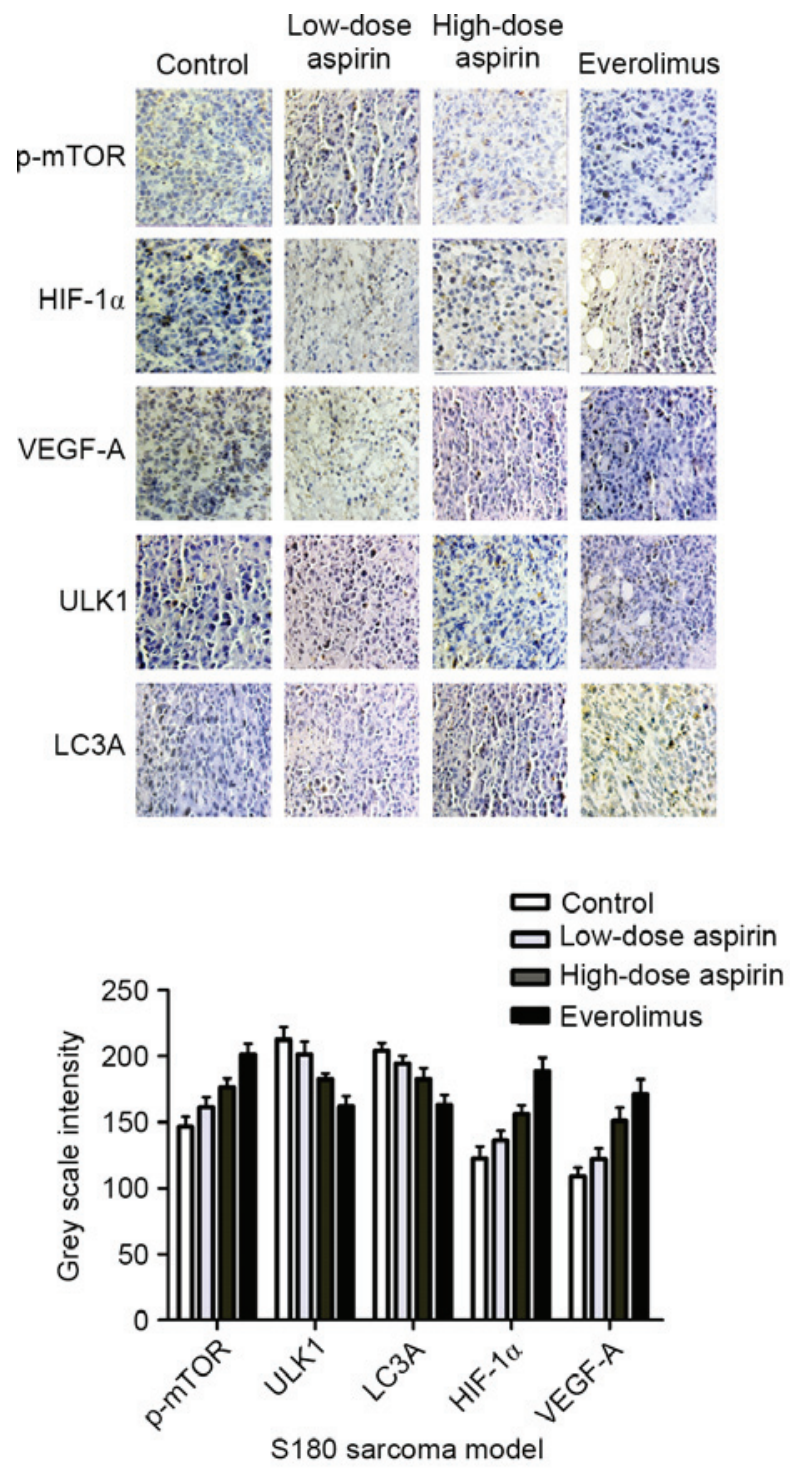

Figure 5. Effects of aspirin on expression of phosphorylated mammalian target of rapamycin (p-mTOR), hypoxia-inducible factor-1 $\alpha$ (HIF-1 $\alpha$ ), vascular endothelial growth factor-A (VEGF-A), UNC-51-like kinase-1 (ULK1) and microtubule-associated protein 1 light chain 3A (LC3A) in S180 sarcoma tissue were detected by immunohistochemisty (original magnification, $\mathrm{x} 400$ ). Gray scale intensity variants were evaluated by Leica Qwin V3 software (original magnification, $\mathrm{x} 400$ ). Three treatment groups were significantly different compared with control, $\mathrm{P}<0.05$.
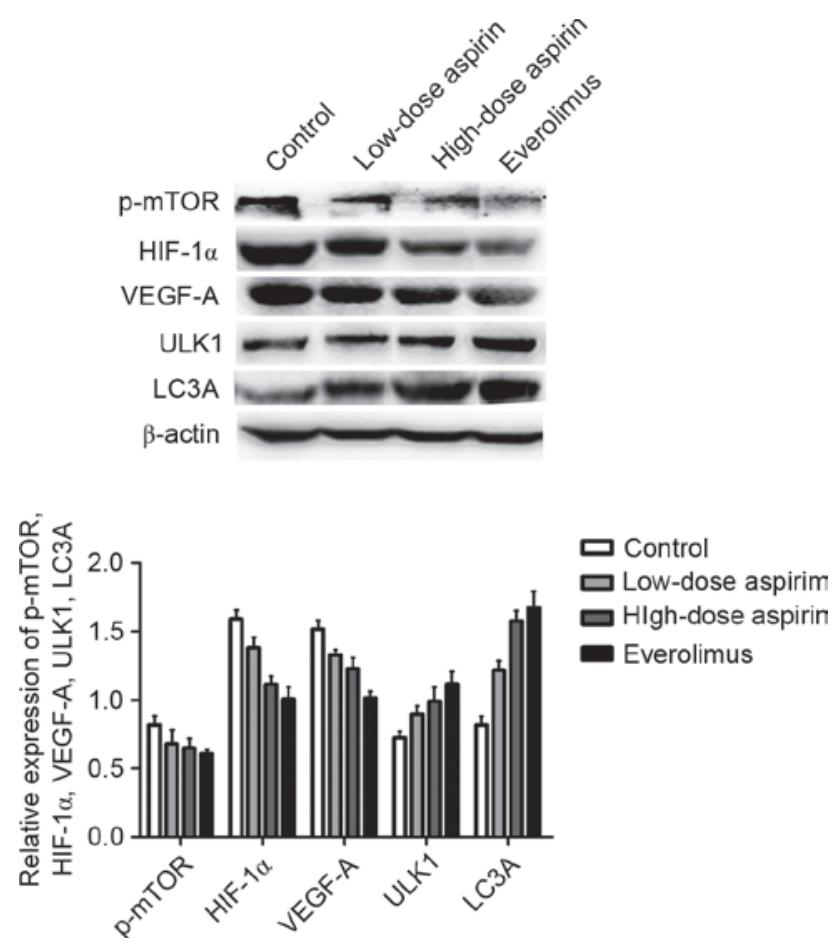

Figure 6. Effects of aspirin on expression of phosphorylated mammalian target of rapamycin (p-mTOR), hypoxia-inducible factor- $1 \alpha$ (HIF-1 $\alpha$ ), vascular endothelial growth factor-A (VEGF-A), UNC-51-like kinase-1 (ULK1) and microtubule-associated protein 1 light chain 3A (LC3A) in S180 sarcoma tissue were detected by western blot analysis. The relative expression of p-mTOR, HIF-1 $\alpha$, VEGF-A, ULK1 and LC3A was analyzed by ImageJ software. In each comparison, there was a significant difference $(\mathrm{P}<0.05)$.

35.6 and $23.4 \%$, respectively (Table II; Fig. 4; $\mathrm{P}<0.05$ for each comparison between treatment groups and the control group). In the low-dose aspirin group and high-dose aspirin group, the mice were in a good general condition, with normal diet and mental state.

Expression of p-mTOR, HIF-1 $\alpha, V E G F-A, U L K 1$ and LC3A in S180 sarcoma tumors. Cells positive for p-mTOR, HIF-1 $\alpha$, VEGF-A, ULK1 and LC3A were stained brown (Fig. 5). The expression of p-mTOR, HIF- $1 \alpha$ and VEGF-A in the control group was markedly higher than that in the treatment groups, but the expression comparison of ULK1, LC3A was the opposite. In each comparison, there was a significant difference $(\mathrm{P}<0.05)$. In addition, aspirin at high concentration and everolimus were significantly different compared with the control group $(\mathrm{P}<0.01)$. As shown in Fig. 5, p-mTOR, HIF-1 $\alpha$ and VEGF-A expression was positively correlated $(\mathrm{r}=0.911$, $\mathrm{P}<0.01 ; \mathrm{r}=0.887 \mathrm{P}<0.01$ ); HIF- $1 \alpha$ and VEGF-A expression was positively correlated $(\mathrm{r}=0.884, \mathrm{P}<0.01) ; \mathrm{p}$-mTOR, ULK1 and LC3A expression was negatively correlated $(r=-0.86, P<0.01$; $\mathrm{r}=-0.856, \mathrm{P}<0.01$ ); and ULK1 and LC3A expression was positively correlated $(\mathrm{r}=0.836, \mathrm{P}<0.01)$.

Effect of aspirin treatment on p-mTOR, HIF-la, VEGF-A, ULK1 and LC3A protein expression as assessed by western blot analysis in S180 sarcoma tumors. P-mTOR, HIF-1 $\alpha$, VEGF-A, ULK1 and LC3A expression was normalized to $\beta$-actin expression by band intensity. As shown in Fig. 6, the expression of p-mTOR, HIF-1 $\alpha$ and VEGF-A decreased in 
the treatment groups, but the expression of ULK1 and LC3A increased when compared with the control group in the S180 sarcoma tissue as with the $\mathrm{H}_{22}$ hepatocarcinoma model. Band intensities were analyzed by ImageJ software, and aspirin at each concentration demonstrated a significant difference between groups $(\mathrm{P}<0.05)$. In the related comparison, $\mathrm{p}-\mathrm{mTOR}$, HIF-1 $\alpha$ and VEGF-A expression was positively correlated $(\mathrm{r}=0.648, \mathrm{P}<0.01 ; \mathrm{r}=0.66, \mathrm{P}<0.01) ; \mathrm{HIF}-1 \alpha$ and VEGF-A expression was positively correlated $(\mathrm{r}=0.907, \mathrm{P}<0.01)$; p-mTOR, ULK1 and LC3A expression was negatively correlated $(\mathrm{r}=-0.621, \mathrm{P}<0.05 ; \mathrm{r}=-0.705, \mathrm{P}<0.01)$; and $\mathrm{ULK} 1$ and LC3A expression was positively correlated $(\mathrm{r}=0.823, \mathrm{P}<0.01)$. The tendency was similar to that observed in the $\mathrm{H}_{22}$ hepatocarcinoma model.

\section{Discussion}

The function of the mTOR signaling pathway as a target of cancer therapy is being actively pursued (27). A study revealed that tumor recurrence was accompanied by strong abnormal expression of rapamycin molecular targets, implying that excessive activation of mTOR signaling pathways was also a significant feature of tumor progression $(28,29)$. Rapamycin and its analogs, including everolimus, which was approved for use as an immunosuppressive agent in transplant patients, have been tested actively in clinical trials for the past few years and have demonstrated preliminary promise of efficacy in several tumor types $(10,30)$. In the present study, we focused primarily on tumor angiogenesis and autophagy effects of drug treatments related to mTOR.

Previous studies have revealed that HIF-1 $\alpha$ expression is necessary for tumor growth in certain tumor cell lines, including hepatomas. Decreased expression of HIF- $1 \alpha$ is associated with slower cell growth and tumor angiogenesis $(12,31)$. HIF-1 $\alpha$ and VEGF-A expression are strongly associated with cancer progression and angiogenesis (12-14). In our study, the control group demonstrated marked expression of p-mTOR, which is the activated form of mTOR and its downstream protein, HIF-1 $\alpha$ and VEGF-A, while the high and low-dose aspirin and everolimus groups effectively inhibited the expression of p-mTOR, HIF- $1 \alpha$ and VEGF-A. To identify and characterize whether aspirin inhibited the overexpression of HIF-1 $\alpha$ and VEGF-A by decreasing p-mTOR expression, we analyzed the expression of p-mTOR, HIF-1 $\alpha$ and VEGF-A by immunohistochemistry and western blotting. The results from the models revealed that p-mTOR, HIF- $1 \alpha$ and VEGF-A expression was positively correlated and decreased in a aspirin dose-dependent manner.

ULK1 as a initiatory protein is regulated by mTOR in the process of autophagy (25). LC3 is a commonly used autophagy marker; its processed form resides in cytoplasm and it could not form without ULK1 (32). mTOR negatively regulates autophagy, and therefore we assessed aspirin's effects on autophagy by immunohistochemistry and western blotting. The results revealed that the expression of $\mathrm{p}$-mTOR decreased in the aspirin groups but ULK1 and LC3A did the opposite. Aspirin may induce autophagy, as evidenced by the increased LC3A. However, in cancer therapy, the role of autophagy is paradoxical. In certain studies, autophagy appeared to function as a protective mechanism against cellular stress $(33,34)$.
However, the induction of autophagy still played a pivotal role in cell death induced by radiations or reagents in other studies (35-37). In the present study, there was an increase in autophagy protein expression and there was clear tissue necrosis. This results suggests that aspirin induces autophagy, leading to an inhibition in tutor growth. This study might form a basis for future studies into the anticancer effects of aspirin.

The inhibitory rates induced by aspirin were 36.7 and $21.1 \%$ in the murine hepatocarcinoma model (Table I and Fig. 1), and 35.6 and $23.4 \%$ in the sarcoma model (Table II and Fig. 4). We also concluded that aspirin reduces tumor growth rates significantly (Figs. 1 and 4).

In the present study, we observed that the levels HIF-1 $\alpha$ and VEGF-A, the downstream proteins of mTOR associated with angiogenesis, decreased in the murine hepatocarcinoma and sarcoma models compared with levels in the non-intervention groups. However, the autophagy-related proteins ULK1 and LC3A were induced, as shown in Figs. 2, 3, 5 and 6. p-mTOR, HIF-1 $\alpha$ and VEGF-A expression was positively correlated, and p-mTOR, ULK1 and LC3A expression was negatively correlated. We have demonstrated that aspirin may inhibit mTOR signaling associated with anti-angiogenesis and promoting autophagy on the protein expression level. We intend to continue with further experiments on the genetic level. Our study has significant clinical reference value and may potentially lead to therapeutic treatment options for hepatoma or sarcoma and other types of cancer.

\section{Acknowledgements}

This study was supported by funding from the National Natural Science Foundation of China (nos. 81073102, 30873408, 81403150 and 81303077).

\section{References}

1. Gee JR, Jarrard DF, Bruskewitz RC, Moon TD, Hedican SP, Leverson GE, Nakada SY and Messing EM: Reduced bladder cancer recurrence rate with cardioprotective aspirin after intravesical bacille Calmette-Guérin. BJU Int 103: 736-739, 2009.

2. Alfonso LF, Srivenugopal KS, Arumugam TV, Abbruscato TJ, Weidanz JA and Bhat GJ: Aspirin inhibits camptothecin-induced p21CIP1 levels and potentiates apoptosis in human breast cancer cells. Int J Oncol 34: 597-608, 2009.

3. Iwama T: NSAIDs and colorectal cancer prevention. J Gastroenterol 44 (Suppl 19): S72-S76, 2009.

4. Din FV, Theodoratou E, Farrington SM, Tenesa A, Barnetson RA, Cetnarskyj R, Stark L, Porteous ME, Campbell H and Dunlop MG: Effect of aspirin and NSAIDs on risk and survival from colorectal cancer. Gut 59: 1670-1679, 2010.

5. Rothwell PM, Wilson M, Elwin CE, Norrving B, Algra A, Warlow CP and Meade TW: Long-term effect of aspirin on colorectal cancer incidence and mortality: 20-year follow-up of five randomised trials. Lancet 376: 1741-1750, 2010.

6. Din FV, Valanciute A, Houde VP, Zibrova D, Green KA, Sakamoto K, Alessi DR and Dunlop MG: Aspirin inhibits mTOR signaling, activates AMP-activatedprotein kinase, and induces autophagy in colorectal cancer cells. Gastroenterology 142: 1504-1515.e3, 2012.

7. Zoncu R, Efeyan A and Sabatini DM: mTOR: from growth signal integration to cancer, diabetes and ageing. Nat Rev Mol Cell Biol 12: 21-35, 2011.

8. Alayev A and Holz MK: mTOR signaling for biological control and cancer. J Cell Physiol 228: 1658-1664, 2013.

9. Guertin DA and Sabatini DM: Defining the role of mTOR in cancer. Cancer Cell 12: 9-22, 2007. 
10. Foster KG, Acosta-Jaquez HA, Romeo Y, Ekim B, Soliman GA Carriere A, Roux PP, Ballif BA and Fingar DC: Regulation of mTOR complex 1 (mTORCI) by raptor Ser863 and multisite phosphorylation. J Biol Chem 285: 80-94, 2010.

11. Carbajo-Pescador S, Ordoñez R, Benet M, Jover R, GarcíaPalomo A, Mauriz JL and González-Gallego J: Inhibition of VEGF expression through blockade of Hifl $\alpha$ and STAT3 signalling mediates the anti-angiogenic effect of melatonin in HepG2 liver cancer cells. Br J Cancer 109: 83-91, 2013.

12. De Francesco EM, Lappano R, Santolla MF, Marsico S, Caruso A and Maggiolini M: HIF-1 $\alpha /$ GPER signaling mediates the expression of VEGF induced by hypoxia in breast cancer associated fibroblasts (CAFs). Breast Cancer Res 15: R64, 2013.

13. Xie SR, Wang Y, Liu CW, Luo K and Cai YQ: Liquiritigenin inhibits serum-induced HIF-1 $\alpha$ and VEGF expression via the AKT/mTOR-p70S6K signalling pathway in HeLa cells. Phytother Res 26: 1133-1141, 2012.

14. Ruan GX and Kazlauskas A: Axl is essential for VEGF-A-dependent activation of PI3K/Akt. EMBO J 31: $1692-1703,2012$.

15. Wouters BG and Koritzinsky M: Hypoxia signalling through mTOR and the unfolded protein response in cancer. Nat Rev Cancer 8: 851-864, 2008.

16. Sengupta S, Peterson TR and Sabatini DM: Regulation of the mTOR complex 1 pathway by nutrients, growth factors, and stress. Mol Cell 40: 310-322, 2010.

17. Jain K, Paranandi KS, Sridharan S and Basu A: Autophagy in breast cancer and its implications for therapy. Am J Cancer Res 3: 251-265, 2013.

18. Morselli E, Galluzzi L, Kepp O, Vicencio JM, Criollo A, Maiuri MC and Kroemer G: Anti- and pro-tumor functions of autophagy. Biochim Biophys Acta 1793: 1524-1532, 2009.

19. Chan EY, Longatti A, McKnight NC and Tooze SA: Kinase-inactivated ULK proteins inhibit autophagy via their conserved C-terminal domains using an Atg13-independent mechanism. Mol Cell Biol 29: 157-171, 2009.

20. Hosokawa N, Sasaki T, Iemura S, Natsume T, Hara T and Mizushima N: Atg101, a novel mammalian autophagy protein interacting with Atg13. Autophagy 5: 973-979, 2009.

21. Kamada Y, Yoshino K, Kondo C, Kawamata T, Oshiro N, Yonezawa K and Ohsumi Y: Tor directly controls the Atg1 kinase complex to regulate autophagy. Mol Cell Biol 30: 1049-1058, 2010 .

22. Behrends C, Sowa ME, Gygi SP and Harper JW: Network organization of the human autophagy system. Nature 466: 68-76, 2010.

23. Wirawan E, Vanden Berghe T, Lippens S, Agostinis P and Vandenabeele P: Autophagy: for better or for worse. Cell Res 22: 43-61, 2012.
24. Moretti L, Yang ES, Kim KW and Lu B: Autophagy signaling in cancer and its potential as novel target to improve anticancer therapy. Drug Resist Updat 10: 135-143, 2007.

25. Goel S, Duda DG, Xu L, Munn LL, Boucher Y, Fukumura D and Jain RK: Normalization of the vasculature for treatment of cancer and other diseases. Physiol Rev 91: 1071-1121, 2011.

26. Zhang X, Wang Z, Wang Z, Zhang Y, Jia Q, Wu L and Zhang W: Impact of acetylsalicylic acid on tumor angiogenesis and lymphangiogenesis through inhibition of VEGF signaling in a murine sarcoma model. Oncol Rep 29: 1907-1913, 2013.

27. Dazert E and Hall MN: mTOR signaling in disease. Curr Opin Cell Biol 23: 744-755, 2011

28. López-Knowles E, O'Toole SA, McNeil CM, Millar EK, Qiu MR, Crea P, Daly RJ, Musgrove EA and Sutherland RL: PI3K pathway activation in breast cancer is associated with the basal-like phenotype and cancer-specific mortality. Int J Cancer 126: 1121-1131, 2010.

29. Hydbring P and Larsson LG: Cdk2: a key regulator of the senescence control function of Myc. Aging (Albany NY) 2: 244-250, 2010

30. Cavazzoni A, Bonelli MA, Fumarola C, La Monica S, Airoud K, Bertoni R, Alfieri RR, Galetti M, Tramonti S, Galvani E, et al: Overcoming acquired resistance to letrozole by targeting the $\mathrm{PI} 3 \mathrm{~K} / \mathrm{AKT} / \mathrm{mTOR}$ pathway in breast cancer cell clones. Cancer Lett 323: 77-87, 2012.

31. Wang FZ, Peng-Jiao, Yang NN, Chuang-Yuan, Zhao YL, Liu QQ, Fei HR and Zhang JG: PF-04691502 triggers cell cycle arrest, apoptosis and inhibits the angiogenesis in hepatocellular carcinoma cells. Toxicol Lett 220: 150-156, 2013.

32. Mizushima N, Yoshimori $T$ and Levine B: Methods in mammalian autophagy research. Cell 140: 313-326, 2010.

33. Chaachouay H, Ohneseit P, Toulany M, Kehlbach R, Multhoff G and Rodemann HP: Autophagy contributes to resistance of tumor cells to ionizing radiation. Radiother Oncol 99: 287-292, 2011.

34. O'Donovan TR, O'Sullivan GC and McKenna SL: Induction of autophagy by drug-resistant esophageal cancer cells promotes their survival and recovery following treatment with chemotherapeutics. Autophagy 7: 509-524, 2011

35. Anbalagan S, Pires IM, Blick C, Hill MA, Ferguson DJ, Chan DA and Hammond EM: Radiosensitization of renal cell carcinoma in vitro through the induction of autophagy. Radiother Oncol 103: 388-393, 2012.

36. Hao J, Pei Y, Ji G, Li W, Feng S and Qiu S: Autophagy is induced by $3 \beta$-Osuccinyl-lupeol (LD9-4) in A549 cells via up-regulation of Beclin 1 and down-regulation mTOR pathway. Eur $\mathrm{J}$ Pharmacol 670: 29-38, 2011.

37. He Z, Mangala LS, Theriot CA, Rohde LH, Wu H and Zhang Y: Cell killing and radiosensitizing effects of atorvastatin in PC3 prostate cancer cells. J Radiat Res 53: 225-233, 2012. 